MY APPROACH

\title{
My approach to the diagnosis of mesothelial lesions
}

\author{
K J Butnor
}

J Clin Pathol 2006;59:564-574. doi: 10.1136/icp.2005.029652

Mesothelial lesions pose considerable diagnostic challenges not only because benign tumours, reactive proliferations and malignant mesothelioma can mimic one another, but also because the morphological patterns displayed by malignant mesothelioma can simulate a variety of epithelial and non-epithelial malignancies. Immunohistochemical markers can aid in distinguishing epithelioid malignant mesothelioma from metastatic adenocarcinoma, but because no single marker reliably separates all cases, a panel of stains is recommended. Immunohistochemical studies are of more limited value in sarcomatoid malignant mesothelioma, and other features often play an essential role. The separation of reactive mesothelial proliferations from malignant mesothelioma on small biopsy can be quite difficult, as distinguishing features, such as stromal invasion, often cannot be adequately assessed. In adequately sampled lesions, however, the distinction between malignant mesothelioma, benign mesothelial proliferations and other tumours can be achieved in most cases by using a carefully intergrated approach that incorporates clinical and radiographic data, immunohistochemical studies and, in selected cases, histochemical and ultrastructural techniques.

Correspondence to: K J Butnor, University of Vermont/Fletcher Allen Health Care, 111 Colchester Avenue, Burlington, VT 05401, USA; kelly.butnor@vtmednet.org

Accepted for publication 7 November 2005
A $\mathrm{n}$ immunohistochemical marker that is both sensitive and specific for malignant mesothelioma remains elusive. Couple this with the broad spectrum of morphological appearances that malignant mesothelioma can exhibit, and it is easy to understand why the diagnosis of malignant mesothelioma is one of the most challenging topics of surgical pathology. This report presents a pattern-based approach to the diagnosis of mesothelial lesions and their mimics and explores the role of immunohistochemistry and other ancillary techniques. The morphological patterns most probably encountered when evaluating suspected mesothelial lesions include epithelioid pattern, spindle cell or sarcomatoid pattern, biphasic pattern and paucicellular or fibrotic pattern.

Table 1 lists the histological patterns seen in malignant mesothelioma.

\section{EPITHELIOID PATTERN}

As the epithelioid variant is the most common histological subtype of malignant mesothelioma and its principal differential consideration, metastatic adenocarcinoma, is the most frequent nonmesothelial neoplasm to involve the serosal membranes, the epithelioid pattern is the most likely pattern to be encountered in cases of suspected malignant mesothelioma.

Epithelioid proliferations of the serosal membranes can take the form of non-descript solid sheets of neoplastic cells. Other architectural arrangements can be more helpful in providing clues to the diagnosis. A pure tubular configuration, in which flattened-to-cuboidal cells encircle hollow spaces, or a tubulopapillary configuration of branching tubules admixed with papillae and trabeculae, favours a diagnosis of epithelioid malignant mesothelioma (EMM; fig 1). Adenomatoid and microcystic configurations, where the microcystic is characterised by cystlike spaces lined by attenuated cells and lakes of basophilic extracellular material, can also be seen in EMM (fig 1). In contrast, true acinar formations composed of columnar cells with eccentric nuclei arranged around a central lumen are not typical of EMM and should suggest a diagnosis of adenocarcinoma. Papillary formations can be seen in both reactive and malignant mesothelial proliferations, as well as in adenocarcinoma (fig 1). Both EMM and adenocarcinoma can also exhibit an adenoid cystic configuration. Such overlap emphasises the need to place architectural features in the context of other findings.

Cytologically, EMM may be deceptively bland. The cells in EMM are typically round, cuboidal or polygonal, and have paracentric nuclei with small nucleoli and a moderate amount of acidophilic cytoplasm (fig 2). Paradoxically, reactive mesothelial lesions often show more cytological atypia than EMM and mitotic figures are generally more abundant. In adenocarcinoma, cells tend to exhibit eccentric nuclei, prominent nucleoli, vesicular chromatin and a greater degree of nuclear overlap and pleomorphism than is seen in malignant mesothelioma (fig 3). Intracytoplasmic vacuoles are seen in both adenocarcinoma and malignant mesothelioma, but unlike the crisp round borders seen in the adenocarcinoma, mesothelial vacuoles are usually degenerative in nature and appear loculated with indistinct borders. Psammoma bodies are occasionally seen in EMMs with a papillary configuration, but when present in abundance, a diagnosis of carcinoma, particularly serous ovarian or primary peritoneal carcinoma, should be strongly favoured. ${ }^{12}$

Abbreviations: $B M M$, biphasic malignant mesothelioma; CEA, carcinoembryonic antigen; DMM, desmoplastic malignant mesothelioma; EMM, epithelioid malignant mesothelioma; SMM, sarcomatoid malignant mesothelioma; TTF-1, thyroid transcription factor-1; HBME-1, human mesothelial Cell-1; WT1, Wilms tumour gene 1 . 

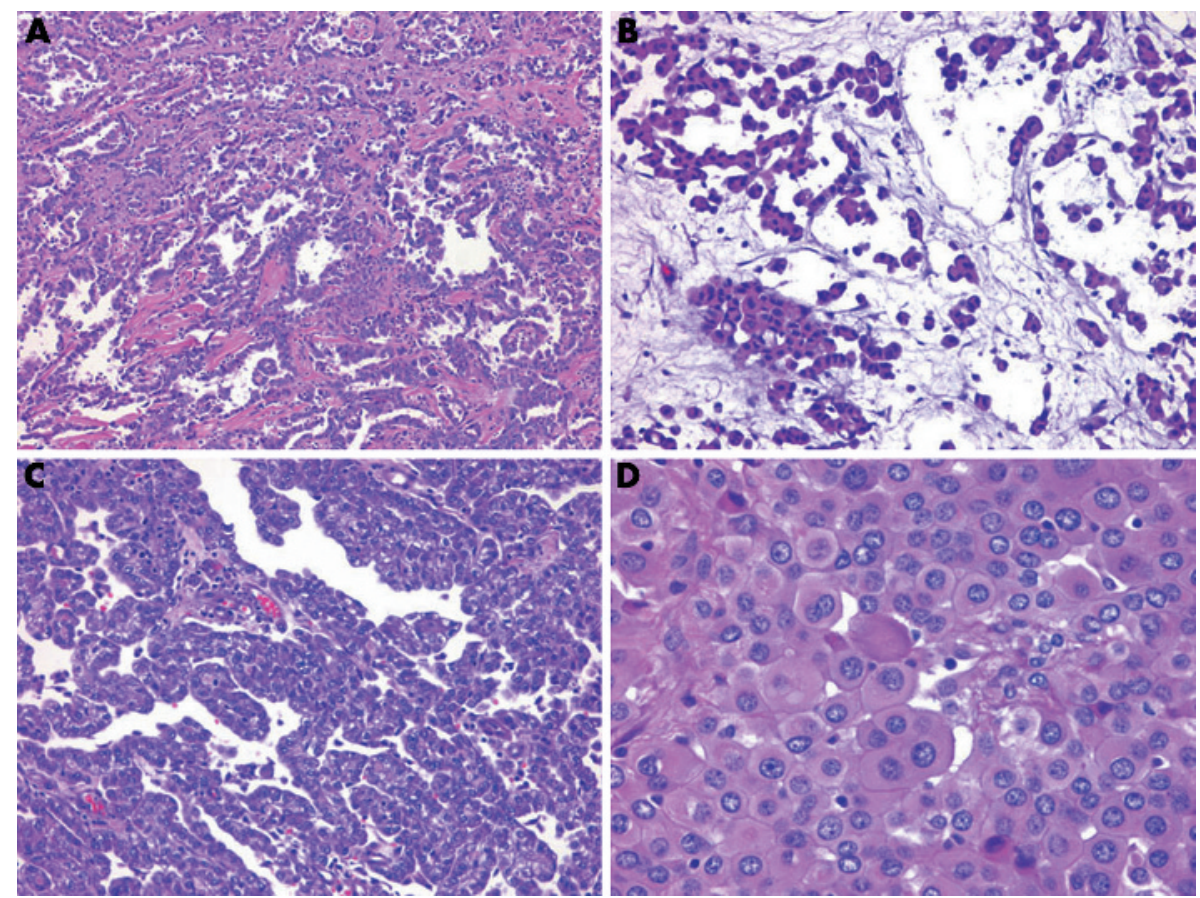

Figure 1 Histological patterns of epithelioid malignant mesothelioma. (A) Tubulopapillary pattern with branching tubules and papillae. (B) Microcystic pattern with lakes of basophilic material. (C) Papillary pattern. (D) Deciduoid pattern with plump polygonal cells with eosinophilic cytoplasm.

These cytological dissimilarities are not a constant feature and EMM may occasionally show quite striking cytological atypia.

\section{Immunohistochemical findings}

The degree of overlap between the morphological appearance of EMM and its mimics warrants the use of ancillary techniques such as immunohistochemical analyses in most cases. As no singularly sensitive and specific immunohistochemical marker for EMM exists, a panel of immunostains is typically used. As there is not a single best panel, a wellconstructed panel includes a pan-cytokeratin, at least two mesothelial markers and at least two carcinoma-associated markers. The choice of antibodies depends largely on which markers show consistently reliable results in the laboratory.

\begin{tabular}{|l|}
\hline Table 1 Histological patterns in malignant \\
mesothelioma \\
\hline Epithelioid \\
Tubular \\
Tubulopapillary \\
Papillary \\
Solid \\
Adenomatoid \\
Adenoid cystic \\
Pleomorphic \\
Deciduoid \\
Clear cell \\
Small cell \\
Sarcomatoid \\
Fibrosarcomatous \\
Osteosarcomatous, chondrosarcomatous, \\
leiomyosarcomatous or rhabdomyosarcomatous \\
Undifferentiated high-grade pleomorphic sarcoma \\
(malignant fibrous histiocytoma)-like \\
Lymphohistiocytoid \\
Biphasic (mixed) \\
Desmoplastic \\
\hline
\end{tabular}

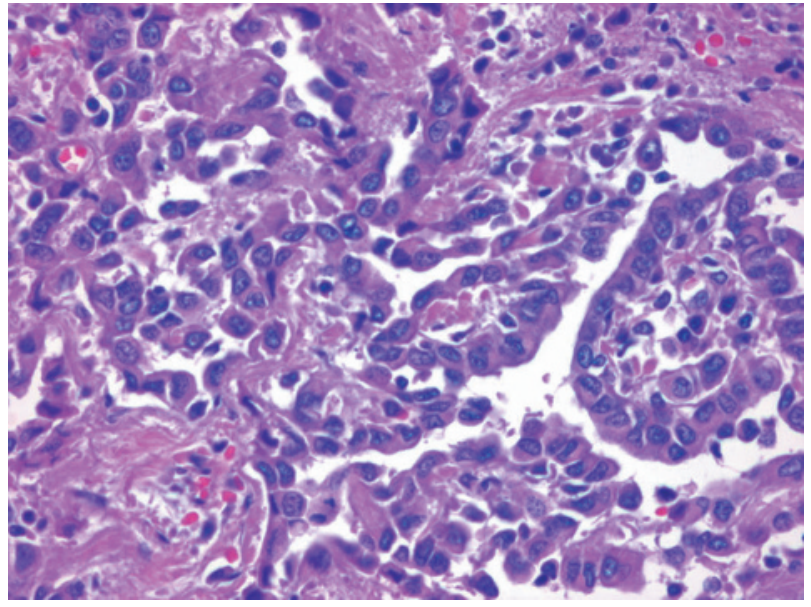

Figure 2 Close-up view of epithelioid malignant mesothelioma, showing cuboidal cells with paracentric, bland nuclei, relatively inconspicuous nucleoli and a moderate amount of cytoplasm.

In my practice, I use a panel comprised of ( 1 ) pan-cytokeratin AEl-AE3/Cam 5.2, (2) cytokeratin 5/6, (3) calretinin, (4) monoclonal carcinoembryonic antigen (CEA) for pleural tumours or B72.3 for peritoneal tumours and (5) thyroid transcription factor-1 (TTF-1) for pleural tumours or Ber-EP4 for peritoneal tumours.

Diffuse staining with pan-cytokeratin is useful because it confirms an epithelial process (fig 4). Cytokeratin 5/6 stains more than $95 \%$ of EMMs (fig 4). ${ }^{3}$ Occasional cytokeratin 5/6 expression has been reported in a variety of adenocarcinomas. ${ }^{3-5}$ Cytokeratin 5/6 expression should be interpreted with caution in cases with a squamoid morphology, as most squamous cell carcinomas are positive. ${ }^{3}$ The sensitivity of calretinin varies depending on the clone used, but is in the range of $73-100 \%{ }^{67}$ Both the nuclear and cytoplasmic compartments of malignant mesothelioma typically stain 


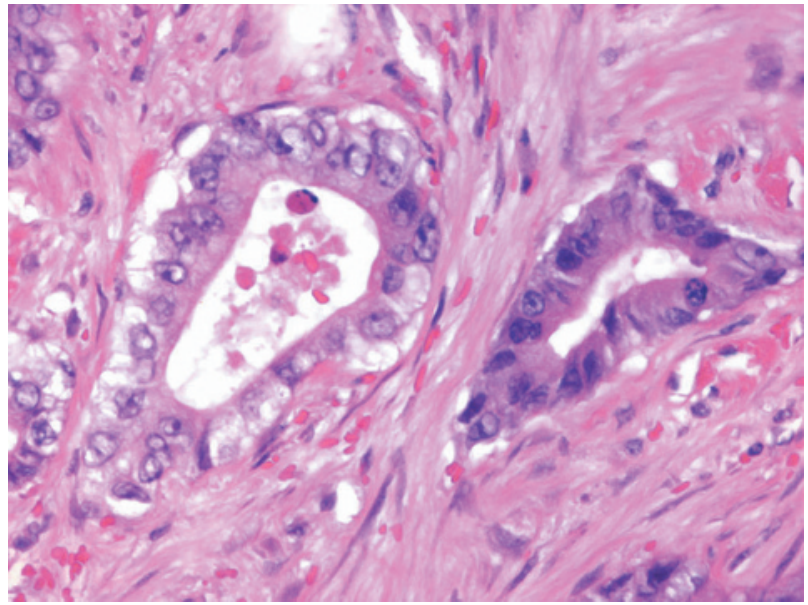

Figure 3 Adenocarcinoma metastatic to the pleura, showing glandforming columnar cells with nucleoli.

with calretinin, although nuclear staining is considered far more specific (fig 4). ${ }^{4}$ Staining limited to the cytoplasm should not be interpreted as unequivocal evidence of mesothelial differentiation, as it can be occasionally seen in carcinomas. ${ }^{6-10}$

Monoclonal CEA serves the dual function of being a reliable negative marker for EMM and a positive marker in most pulmonary adenocarcinomas (fig 5). ${ }^{11}$ Monoclonal CEA is preferred to polyclonal CEA because polyclonal CEA has a propensity for non-specific background staining. ${ }^{12}$ For pleural biopsies, a second sensible carcinoma-associated marker is TTF-1. Thyroid transcription factor-1 shows nuclear staining in $75 \%$ or more of pulmonary adenocarcinomas and is consistently negative in EMM (fig 5). ${ }^{13}{ }^{14}$ Cytoplasmic TTF- 1 staining is non-specific and has been reported in carcinomas from several sites. ${ }^{15}$
Although CEA is a sensitive marker for pulmonary adenocarcinoma, it detects $<20 \%$ of primary peritoneal or ovarian serous carcinomas. ${ }^{16}$ A more suitable choice for peritoneal biopsies is B72.3, which diffusely stains around $70-80 \%$ of adenocarcinomas, including ovarian carcinoma, and is negative in most EMMs. ${ }^{10}$ As thyroid transcription factor-1-positive tumours do not generally enter the differential diagnosis of peritoneal tumours, Ber-EP4 can be substituted as a second carcinoma-associated marker in peritoneal biopsies. Similar to B72.3, Ber-EP4 is diffusely positive in a high proportion of adenocarcinomas. ${ }^{17}$ Ber-EP4 staining occurs in a minority of malignant mesotheliomas and when present, is typically restricted to a few cells. ${ }^{8}{ }^{17} 18$

In tumours that are positive for only one of the two mesothelial-associated or carcinoma-associated markers, only pan-cytokeratin-positive, or show conflicting results, additional markers may be helpful (table 2). The newly developed markers D2-40 and anti-podoplanin show membranous staining in 86-96\% of EMMs (fig 4). ${ }^{19} 20$ Both antibodies have been recently shown to recognise the same protein, the human mucin-type glycoprotein podoplanin..$^{21}$ Carcinomas from several sites, such as lung, breast and kidney, are negative for these markers. D2-40, however, must be used with caution in peritoneal biopsies, as staining was observed in some ovarian serous carcinomas. ${ }^{19}$ As D2-40 and podoplanin also highlight lymphatic endothelial cells, care must be taken not to misinterpret staining of lymphatic channels as evidence of mesothelial differentiation. The list of vascular tumours that are recognised by these markers continues to grow. The Wilms tumour gene 1 protein (WT1) shows nuclear staining in about of $80 \%$ of EMMs. ${ }^{142-24}$ Although this is a useful marker in pleural biopsy, in which the main distinction is between EMM and pulmonary adenocarcinoma, it is of limited utility in peritoneal biopsies, as a substantial number of renal, ovarian and endometrial carcinomas stain for this marker. ${ }^{25-27}$ Thrombomodulin is also positive in a high proportion of EMM, but like cytokeratin $5 / 6$, this marker stains most squamous cell carcinomas and,

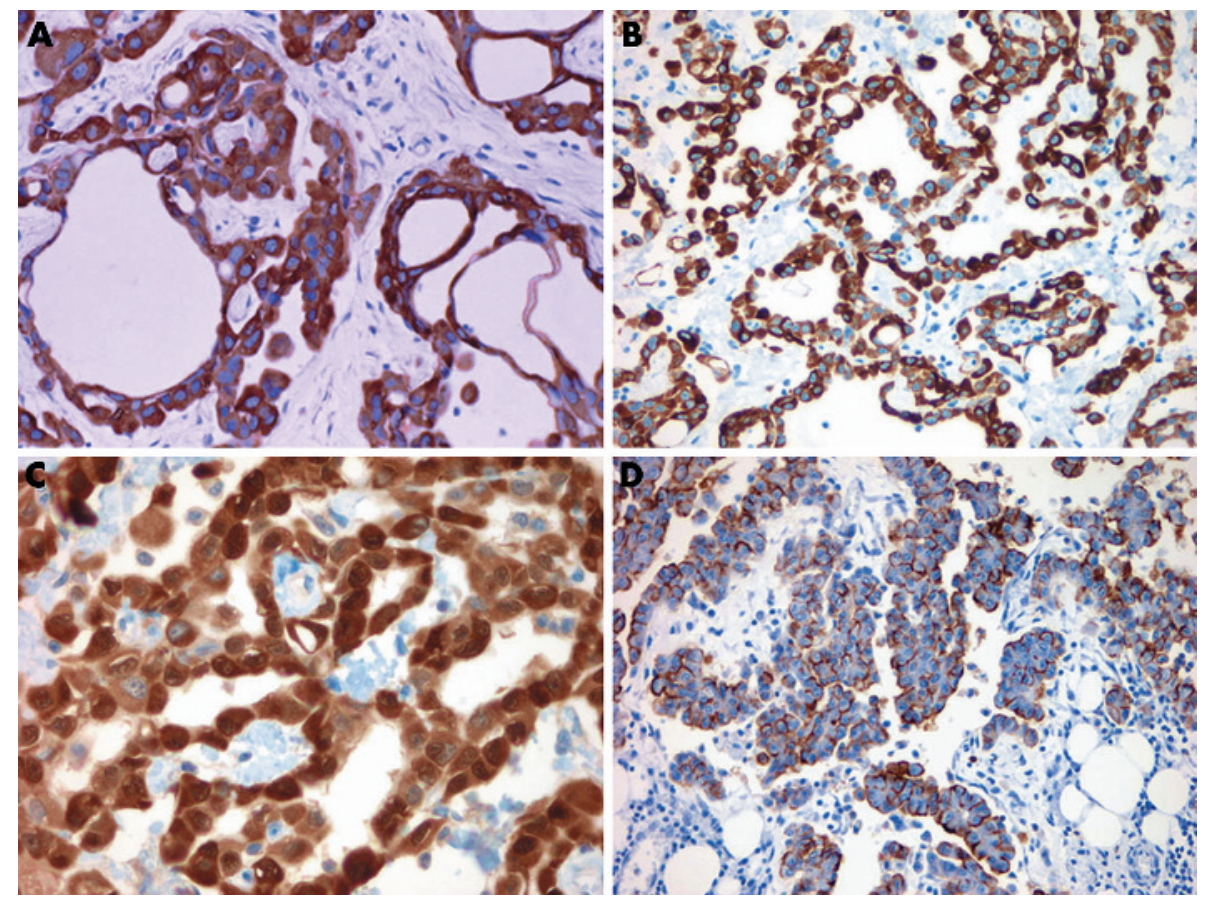

Figure 4 Immunohistochemical staining in epithelioid malignant mesothelioma. (A) Diffuse, strong staining for pan-cytokeratin. (B) Cytoplasmic cytokeratin 5/6 staining. (C) Calretinin stains both the nuclei and cytoplasm. (D) D2-40 exhibits a membranous staining pattern. Case (D) a kind giff from James Burchette, Duke University, Durham, North Carolina, USA. 


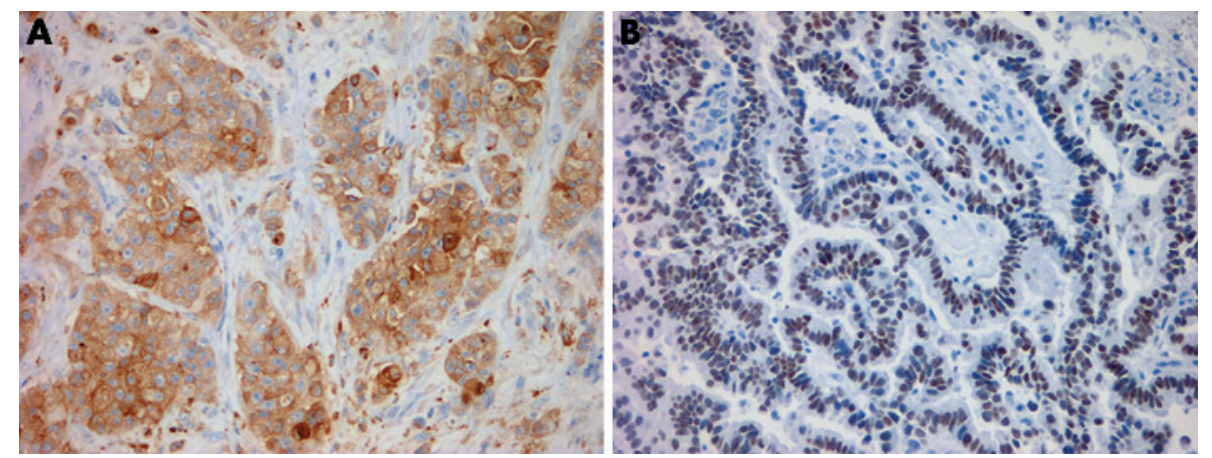

Figure 5 Immunohistochemical staining of lung adenocarcinoma metastatic to the pleura. (A) Monoclonal carcinoembryonic antigen. (B) Thyroid transcription factor- 1 .

in addition, is often positive in urothelial and renal cell carcinomas. ${ }^{28-30}$ Despite initial enthusiasm, human mesothelial cell-1 (HBME-1), mesothelin and an array of other purported mesothelial markers have subsequently been shown to lack specificity for EMM. ${ }^{31-33}$

Additional exclusionary markers for EMM include MOC31, which diffusely stains a very high proportion of carcinomas and is only focally immunoreactive in rare EMMs, and BG-8, which shows comparable results. ${ }^{10} 34$ CD15 (Leu-Ml) stains around $60-70 \%$ of adenocarcinomas and is negative in most EMMs, with most of the rare positive cases occurring in the peritoneum. ${ }^{1}{ }^{10} 323536$

\section{Histochemical findings}

With the proliferation of sophisticated immunohistochemical markers, interest in histochemical stains for the diagnosis of malignant mesothelioma has waned. Histochemical techniques, however, should not be overlooked, particularly when immunostains produce conflicting or equivocal results. Intense staining of intracytoplasmic vacuoles or luminal secretions for periodic acid-Schiff after pretreatment with

Table 2 Expected immunohistochemical and histochemical staining reactions in epithelioid malignant mesothelioma and adenocarcinoma

\begin{tabular}{lll}
\hline Marker/stain & Mesothelioma & Adenocarcinoma \\
\hline Pan-cytokeratin & + & + \\
Cytokeratin 5/6 & + & $-{ }^{*}$ \\
Calretinin & + & - \\
CEA & - & + \\
B72.3 & - & + \\
TTF-1 & - & $+\dagger$ \\
Ber-EP4 & - & + \\
D2-40 & + & - \\
WT1 & + & $-\ddagger$ \\
Thrombomodulin & + & $-\S$ \\
MOC-31 & - & + \\
BG-8 & - & + \\
CD15 (Leu-M1) & - & + \\
Periodic acid-Schiff & + or - & + \\
without diastase & & \\
Periodic acid-Schiff & - & + \\
with diastase (PAS-D) & & \\
Alcian blue & + & If + without digestion, \\
Alcian blue with & - & remains + after digestion \\
hyaluronidase & &
\end{tabular}

+ , positive; -, negative

*Positive in a small proportion of non-pulmonary adenocarcinomas. †Nuclear staining in pulmonary adenocarcinoma and thyroid carcinoma.

$\ddagger$ Positive in some renal, ovarian and endometrioid carcinomas. $\S$ Positive in most renal and urothelial carcinomas.

CEA, Monoclonal carcinoembryonic antigen; TTF-1 thyroid transcription factor-1; WT1, Wilms tumour gene 1; PAS-D, periodic acid-Schiff-D. diastase or Alcian blue after hyaluronidase, which is indicative of neutral acid mucin production, favours a diagnosis of adenocarcinoma, whereas absent or markedly decreased staining after pretreatment is more consistent with malignant mesothelioma (table 2; fig 6). ${ }^{37}$ Mucicarmine should not be used, as it occasionally cross reacts with hyaluronic acid. Histochemical stains are generally not useful for the diagnosis of non-epithelioid malignant mesotheliomas.

\section{Ultrastructural findings}

When immunohistochemical and histochemical results are inconclusive, detecting characteristic ultrastructural features by electron microscopy may facilitate the diagnosis of malignant mesothelioma. Although a detailed discussion of the ultrastructure of malignant mesothelioma is outside the scope of this review, a key ultrastructural element of EMM is the presence of long, slender microvilli. ${ }^{38}{ }^{39}$ Unfortunately, distinguishing features may not be present in all cases, particularly in poorly differentiated tumours. ${ }^{40}$

\section{Differential considerations}

The main entity to exclude when considering a diagnosis of EMM is metastatic carcinoma; ancillary techniques provide considerable support in this distinction. Accurate diagnosis, however, requires the integration of clinicoradiographic information. In some instances, there is a pre-existing diagnosis of carcinoma at an extraserosal site. Comparison of the histological features of a serosal biopsy specimen with those of the primary tumour may obviate the need for immunohistochemical stains or prompt the use of sitespecific carcinoma-associated markers, such as thyroglobulin or prostate specific antigen.

It is also essential to confirm that the gross distribution of tumour is compatible with the histological impression. In cases of metastatic carcinoma, an extraserosal mass is usually apparent and the serosa is typically studded by multiple small nodules or is irregularly thickened by discrete masses. By contrast, malignant mesothelioma usually features confluent nodules or forms a diffuse thick rind-like growth that encases the subjacent organs. Not all tumours that diffusely involve the serosa, however, represent malignant mesothelioma, nor are all malignant mesotheliomas diffusely distributed. Rarely, carcinomas and other non-mesothelial tumours can exhibit a growth pattern similar to that of malignant mesothelioma, which has been termed pseudomesotheliomatous. ${ }^{41-43}$ Malignant mesothelioma uncommonly manifests as a sharply circumscribed serosal tumour. ${ }^{44}$ Localised malignant mesothelioma seems to have a much better prognosis than the diffuse form of malignant mesothelioma. ${ }^{45}$

Several rare variants of EMM deserve special mention because of their propensity to be confused with other 

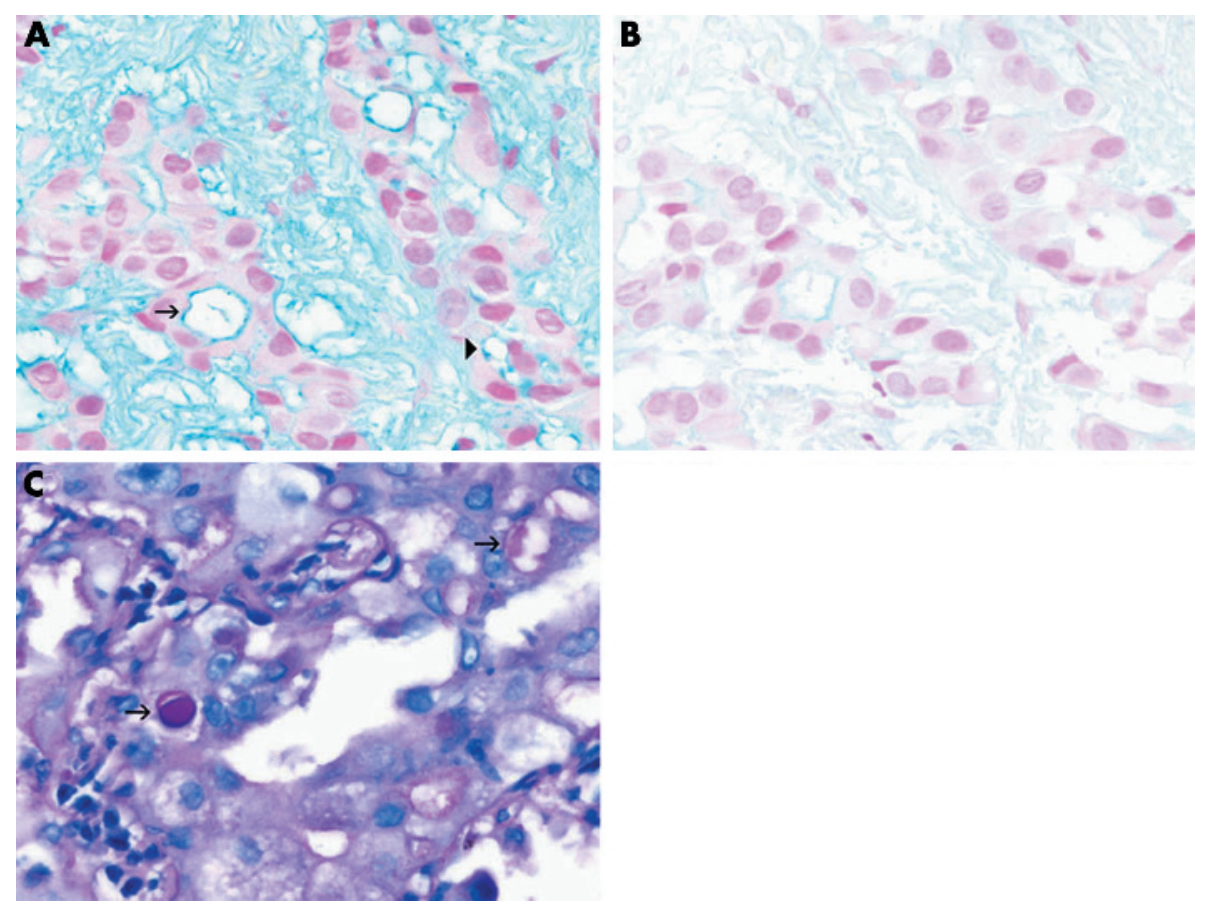

Figure 6 (A) Alcian blue staining of epithelioid malignant mesothelioma highlights intracytoplasmic (arrowhead) and intraluminal (arrow) droplets of hyaluronic acid. (B) Staining is markedly reduced in a serial section pretreated with hyaluronidase. (C) Periodic acid-Schiff staining of neutral mucin is unaffected by diastase digestion, as is shown in the cytoplasmic vacuoles (arrows) of this adenocarcinoma metastatic to the pleura.

tumours. Clear cell malignant mesothelioma can histologically mimic conventional renal cell carcinoma. ${ }^{46}$ Distinguishing renal cell carcinoma from clear cell malignant mesothelioma can be quite challenging, not only because renal cell carcinoma has the propensity to metastasise long before the primary tumour is discovered but also because renal cell carcinoma does not consistently stain with some carcinoma-associated markers, such as CEA. The most reliable markers for distinguishing clear cell malignant mesothelioma from renal cell carcinoma seem to be calretinin, cytokeratin 5/6 and CD15. ${ }^{47} 48$

Deciduoid malignant mesothelioma is comprised of sheets of plump round-to-polygonal cells with eosinophilic cytoplasm (fig 1). Deciduoid malignant mesothelioma has been confused with ectopic decidual reaction and may also be

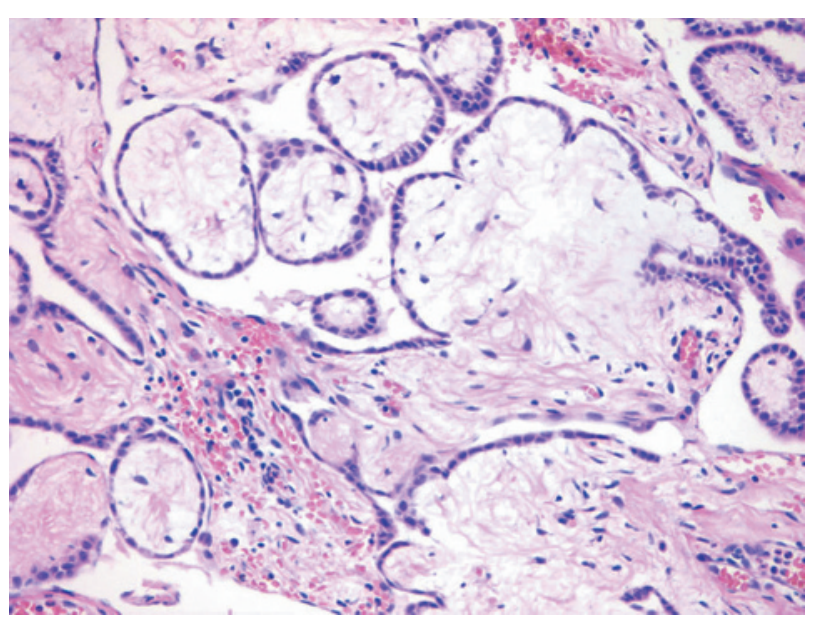

Figure 7 Well-differentiated papillary mesothelioma with broad fibrovascular cores lined by a single layer of bland cuboidal mesothelial cells. mistaken for other oncocytoid tumours, such as hepatocellular carcinoma and adrenocortical carcinoma. ${ }^{49}$ Hep-Parl and inhibin, markers that are positive in hepatocellular carcinoma and adrenocortical carcinoma, respectively, are negative in EMM. ${ }^{50}$

EMM with a predominantly papillary configuration must be distinguished not only from adenocarcinomas such as ovarian and primary peritoneal serous carcinoma but also from well-differentiated papillary mesothelioma, a generally indolent tumour characterised by serosal plaques or small nodules composed histologically of fibrovascular cores lined by bland cuboidal mesothelial cells (fig 7). Although it is debatable whether focal invasion is acceptable in welldifferentiated papillary mesothelioma, tumours with diffuse

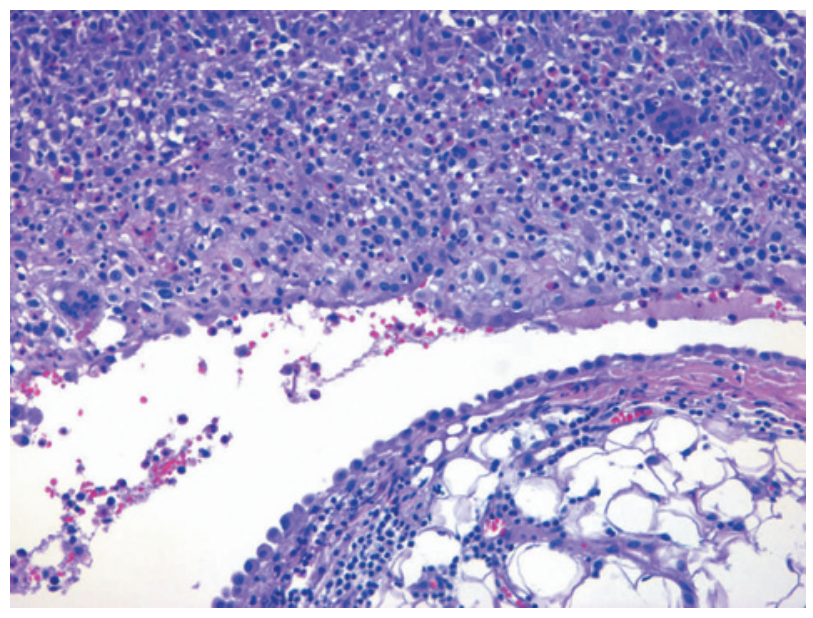

Figure 8 Exfoliated reactive mesothelium sometimes forms densely packed sheets within the peritoneum cavity or pleural space (top). This case is from a patient with spontaneous pneumothorax. Stromal invasion is absent. 

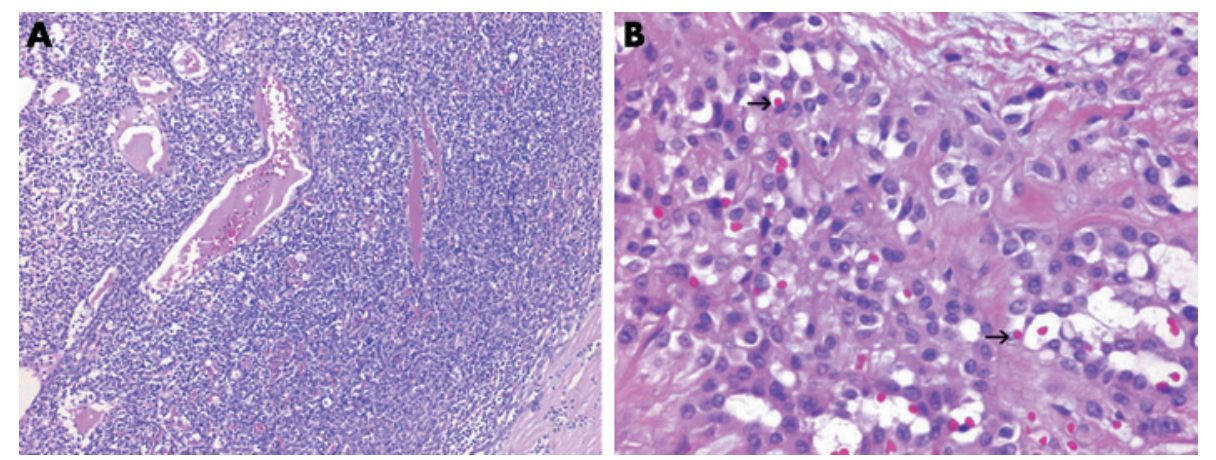

Figure 9 (A) Thymoma directly extending the pleura showing an organoid growth pattern and perivascular lakes. (B) Epithelioid haemangioendothelioma of the pleura composed of plump epithelioid cells, some of which feature intracytoplasmic lumens with red blood cells (arrows).

invasion should be designated as EMM. ${ }^{51-55}$ The superficial portion of some EMMs can exhibit a well-differentiated papillary mesothelioma-like pattern, which can create diagnostic difficulties in small biopsy specimens in which stromal invasion cannot be definitively assessed.

Diffuse stromal invasion is also helpful in differentiating EMM from reactive mesothelial proliferations, although care must be taken not to overinterpret reactive mesothelial cells entrapped within granulation tissue or organising serositis as true stromal invasion..$^{56}$ Sheets of mesothelial cells packed within the pleural or peritoneal space seen in reactive conditions and without stromal invasion, should not be diagnosed as malignant (fig 8). Occasionally, nodular proliferations along the serosal membranes are actually composed of histiocytes rather than mesothelial cells, which is termed nodular histiocytic hyperplasia. ${ }^{57}$ Immunohistochemically, EMA and p53 seem to be preferentially expressed in malignant mesothelioma, whereas desmin immunoreactivity is more common in the reactive mesothelium..$^{58}$ As overlap exists, however, the US-Canadian Mesothelioma Reference Panel recommends that these markers not be routinely used in individual cases. ${ }^{56}$ The distinction of reactive pleuritis from malignant mesothelioma is discussed later (see desmoplastic malignant mesothelioma).

Benign tumours of mesothelial origin also have the potential to pose diagnostic problems in small biopsy samples. Adenomatoid tumours are composed of flat-tocuboidal cells arranged in cords and tubules, but unlike most EMMs, are solitary, sharply circumscribed lesions. Multiloculated peritoneal inclusion cysts, or benign multicystic mesothelioma, typically feature multiple translucent peritoneal cysts lined by bland flattened mesothelium. ${ }^{60}$ The gross appearance and lack of stromal invasion distinguishes this entity from the microcystic variant of EMM.

Thymoma, which can be metastatic to or arise primarily in the pleura, variably expresses antimesothelial antibodies. ${ }^{61}$
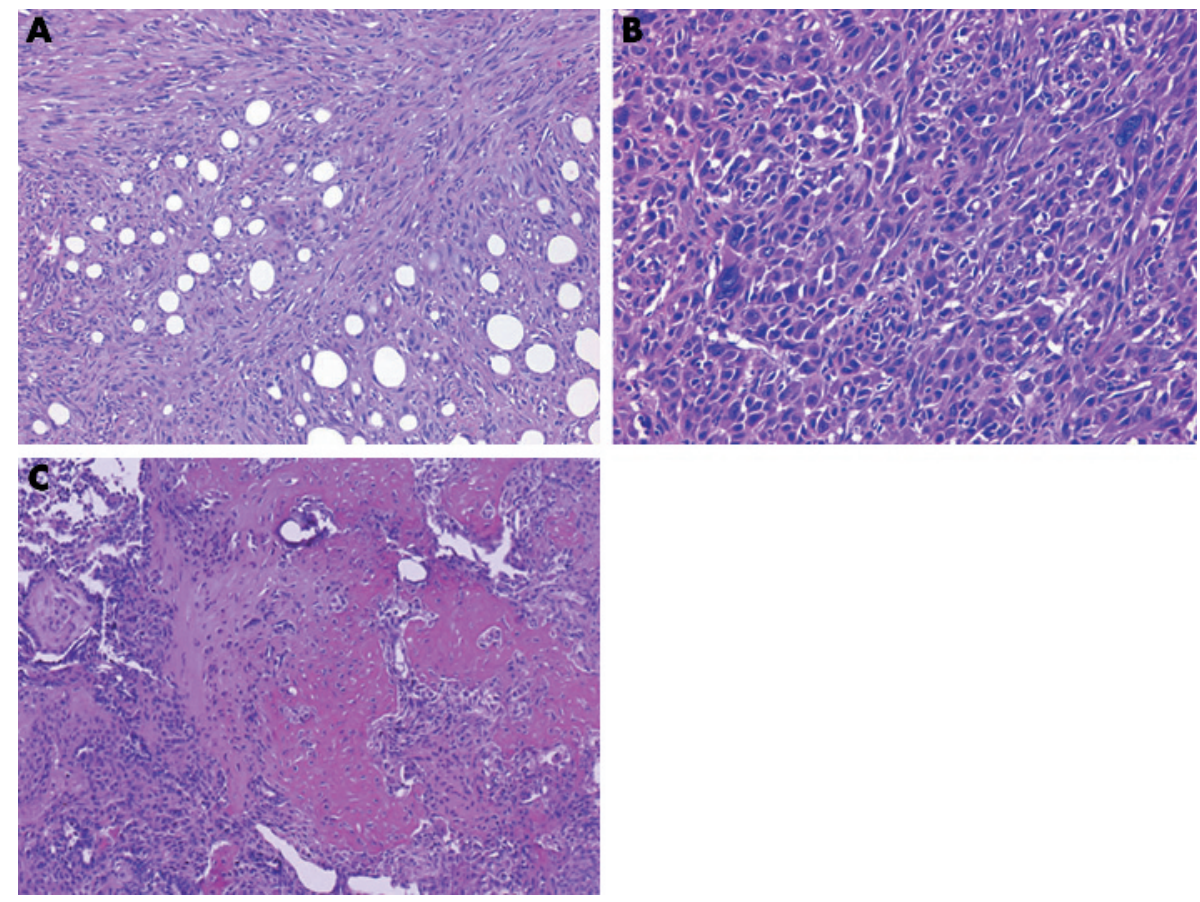

Figure 10 Histological spectrum of sarcomatoid malignant mesothelioma. (A) Spindle cells with a storiform arrangement resembling fibrosarcoma. (B) Malignant fibrous histocytoma-like pattern featuring anaplastic and bizarre multinucleated tumour cells. (C) Heterologous elements are sometimes present, as in this sarcomatoid malignant mesothelioma with osteoid differentiation. 
The possibility of thymoma should be considered in tumours with a lobulated architecture, perivascular lakes or organoid growth (fig 9). The expression of immature lymphocytes by terminal deoxynucleotide transferase (TdT) or CDla staining can further help in the distinction from EMM. Germ cell tumours occasionally involve the serosa, but are negative for calretinin and stain for placental alkaline phosphatase. ${ }^{62}{ }^{63}$ Vascular tumours, such as epithelioid hemangioendothelioma and angiosarcoma, rarely involve the pleura (fig 9). Although they may show aberrant cytokeratin expression, in contrast with that in EMM, staining is typical patchy or weak, and expression of mesothelial-associated markers, such as calretinin and cytokeratin 5/6, is absent. ${ }^{6465}$ The vascular nature of these tumours can be highlighted by staining for CD31, CD34 and factor VIII. Other non-epithelial tumours that can mimic EMM include malignant melanoma and lymphoma, which can be excluded by diffuse staining for pan-cytokeratin.

\section{SPINDLE CELL OR SARCOMATOID PATTERN}

Occasionally, tumours involving the serosal membranes feature a spindle cell or frankly sarcomatoid pattern. Sarcomatoid malignant mesotheliomas (SMMs), which constitute about $15-20 \%$ of malignant mesotheliomas, range from fibroblast-like spindle cells arranged in a storiform, fascicular or haphazard pattern reminiscent of fibrosarcoma to malignant fibrous histiocytoma-like tumours with anaplastic and sometimes multinucleated cells (fig 10). ${ }^{66}$ Rarely, SMMs show leiomyoid, chondroid, osseous or rhabdomyoblastic differentiation (fig 10). ${ }^{37}$ 67-69

\section{Immunohistochemical findings}

The mesothelial markers so often useful in the diagnosis of EMM are of limited value in SMM. Calretinin immunoreactivity is seen in only $39-70 \%$ of SMMs, whereas cytokeratin $5 / 6$ stains $0-29 \%$ of cases. ${ }^{70}$ Spindle cell neoplasms that mimic SMM occasionally stain for antimesothelial antibodies. ${ }^{70}{ }^{71}$ Mesenchymal differentiation can sometimes be shown immunohistochemically in SMMs in the form of smooth muscle actin, desmin or S-100 expression. ${ }^{70} 72$

Because of the inconsistent staining of SMMs for mesothelial markers, in cases of suspected SMM, I generally carry out staining with a limited panel consisting of pancytokeratin and vimentin. Expression of vimentin, which is present in most malignant mesotheliomas, assures that the tissue has undergone proper fixation. ${ }^{39}$ Pan-cytokeratin stains around $\geqslant 70 \%$ SMMs, often intensely and diffusely. ${ }^{70}{ }^{71}$ In contrast, most sarcomas are non-immunoreactive or only focally positive.

\section{Ultrastructural findings}

SMMs often lack the more helpful features generally associated with malignant mesotheliomas and instead ultrastructurally resemble soft tissue fibrosarcomas. ${ }^{37}$

\section{Differential considerations}

SMMs, especially those displaying heterologous elements, may simulate sarcomas. A wide variety of sarcomas can metastasise or directly invade the serosa from adjacent organs and a few sarcomas may arise as primary serosal tumours. Although pan-cytokeratin immunoreactivity usually helps in the distinction, the separation of SMM from sarcoma requires the integration of clinical and radiographic information. Serosal involvement by metastatic sarcoma is typically a late manifestation. Comparison of the histological features of the serosal and primary tumours can facilitate diagnosis.

It is more difficult to distinguish SMM from sarcomatoid carcinoma, as pan-cytokeratin cannot be used as a

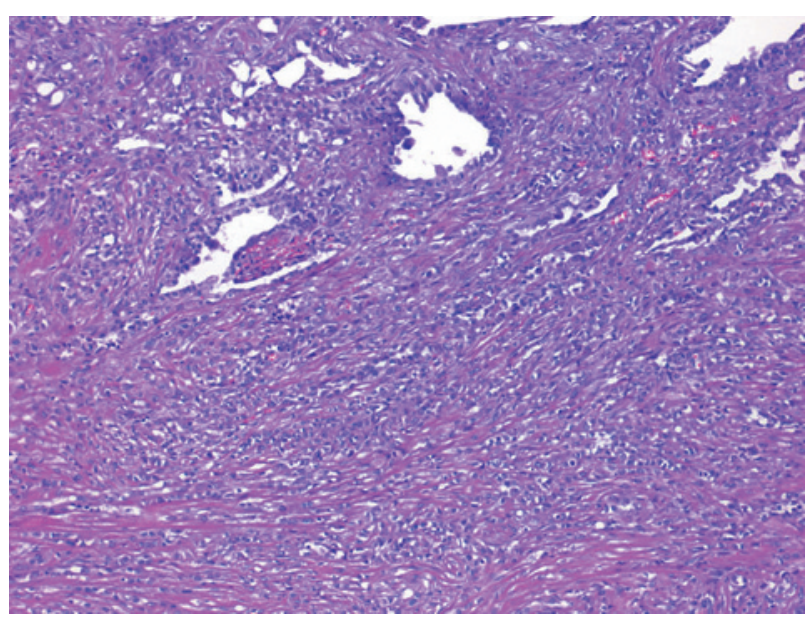

Figure 11 Biphasic malignant mesothelioma showing an epithelioid component comprising of tubules (top) merging with a sarcomatoid component of spindled cells arranged in fascicles (bottom).

discriminator. As with sarcomas, careful attention to whether there is radiographic evidence of an extraserosal mass is essential. As for EMM, malignant melanoma, particularly the spindle cell variant, is in the differential for SMM.

\section{BIPHASIC PATTERN}

The biphasic pattern, although less common than the epithelioid pattern, is more common than a purely sarcomatoid pattern in serosal biopsies. About 30\% of malignant mesotheliomas exhibit both epithelioid and sarcomatoid components (fig 11)..$^{54}$ The minor component should comprise $>10 \%$ of the tumour to be designated as biphasic, or mixed-type, malignant mesothelioma (BMM). ${ }^{54}$ The detection of a sarcomatoid component in an otherwise epithelioid tumour, or vice versa, is dependent on the extent of sampling, as shown by a recent study in which $20 \%$ of tumours diagnosed as EMM at pleural biopsy were reclassified as BMM at extrapleural pneumonectomy. ${ }^{73}$ In some cases, there is a gradual transition between epithelioid and sarcomatoid components, although others are sharply demarcated.

As may be expected, the utility of ancillary techniques in BMM falls between that which is reported for EMM and SMM. It is not uncommon for BMMs to show strong immunoreactivity for mesothelial markers in the epithelioid component and weak or absent staining in the sarcomatoid component.

\section{Differential considerations}

BMM must be distinguished from metastatic pleomorphic carcinoma from several sites, most commonly from the lung. This distinction can usually be achieved by assessing the immunostaining characteristics of the epithelioid areas of a tumour for mesothelial-associated and carcinoma-associated markers. Non-pleomorphic carcinomas metastatic to the serosa can incite such an exuberant desmoplastic response as to also simulate BMM. Other considerations include metastatic malignant melanoma and biphasic synovial sarcoma. Biphasic synovial sarcoma can be metastatic or occur as a primary tumour of the pleura (fig 12). Synovial sarcoma often stains at least focally for pan-cytokeratin and calretinin, but unlike BMM, is usually at least focally positive for Ber-EP4, negative for thrombomodulin and negative for WT1. ${ }^{71} 74$ BMM lacks the $(\mathrm{X} ; 18)$ chromosomal translocation that can be detected through molecular techniques in synovial sarcoma. ${ }^{75}$ 


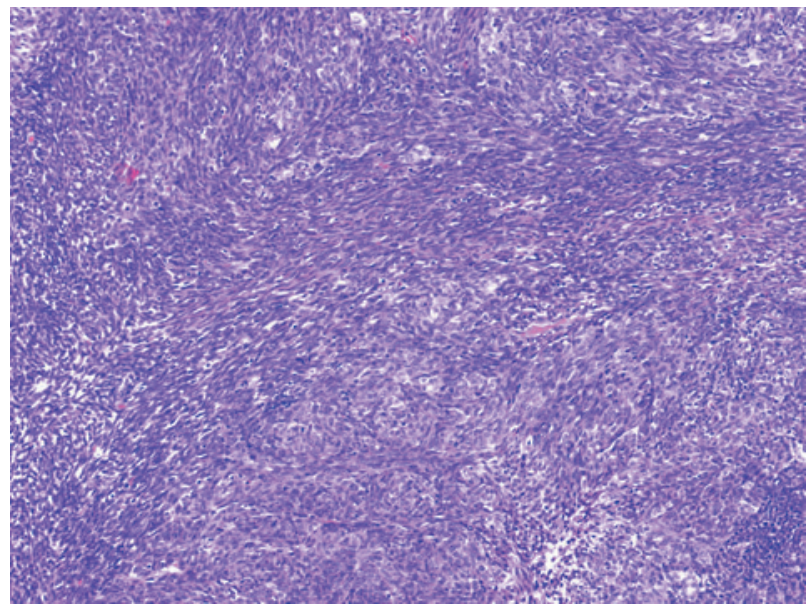

Figure 12 Biphasic synovial sarcoma metastatic to the pleura showing epithelioid cells interspersed with sarcomatoid foci.

\section{PAUCICELLULAR OR FIBROTIC PATTERN}

The paucicellular or fibrotic pattern is arguably the most challenging pattern to evaluate in serosal biopsy samples because of the potential for misdiagnosing benign processes as malignant and, conversely, malignant tumours as benign. Desmoplastic malignant mesothelioma (DMM) is an underrecognised variant of malignant mesothelioma that can be deceptively bland. This subtype, which accounts for $5-10 \%$ of malignant mesotheliomas, most commonly affects the pleura. $^{76-78}$ Its banal appearance belies the aggressive nature of this tumour.

DMMs are characterised by dense paucicellular hyalinised collagen amid which spindle or stellate tumour cells, often associated with slit-like spaces, are arranged in a storiform or patternless arrangement (fig 13). ${ }^{76}{ }^{79}$ This appearance should comprise at least $50 \%$ of a tumour to be designated as DMM. ${ }^{54}$ Sarcomatoid foci are usually present, but may be quite small and difficult to detect without adequate sampling. Epithelioid foci can be occasionally seen. The presence of frankly sarcomatoid areas, in conjunction with one or more of the following, is considered highly specific for DMM: bland infarct-like necrosis, invasion of chest wall
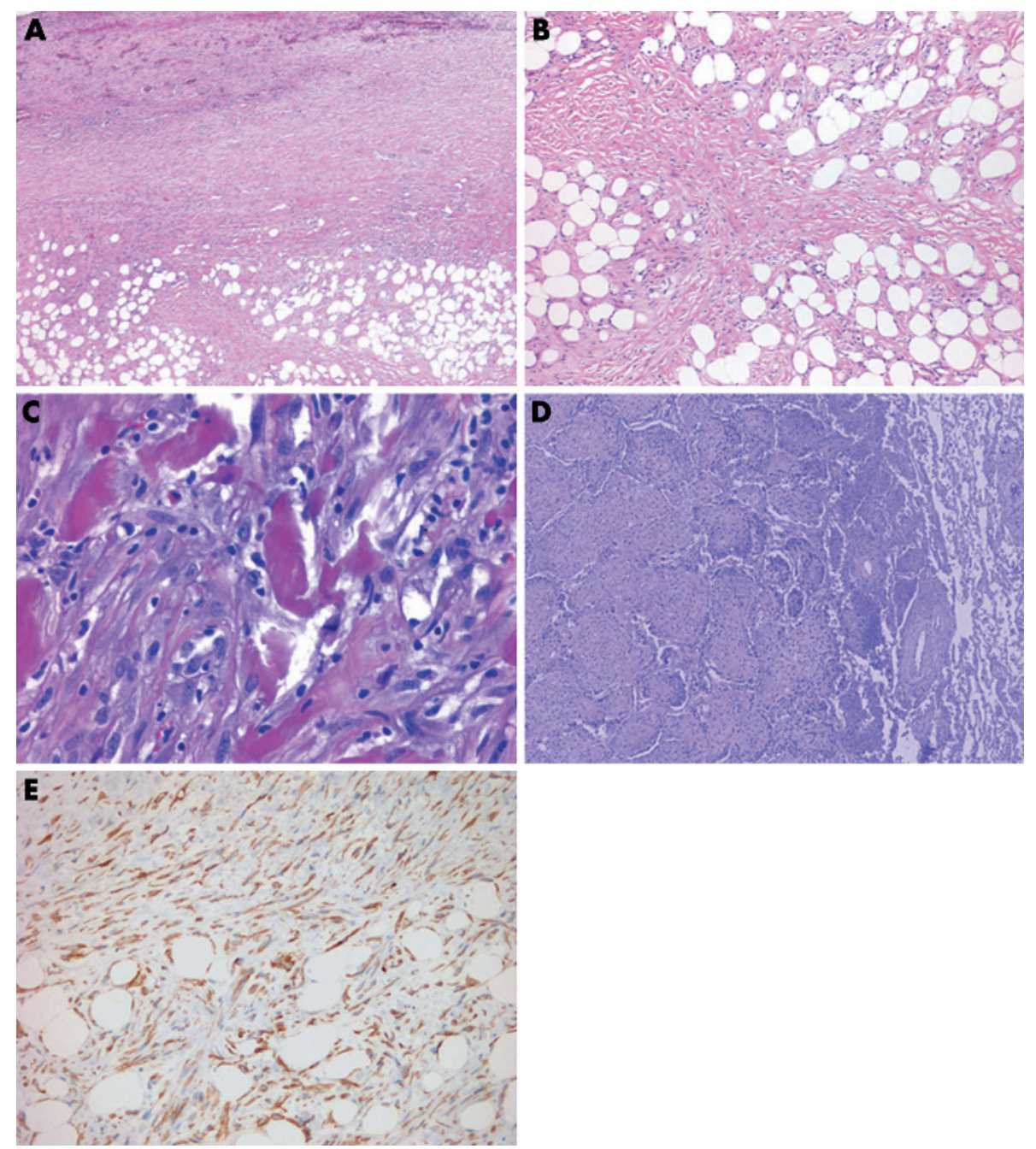

Figure 13 (A) At low magnification, this desmoplastic malignant mesothelioma appears deceptively bland with features reminiscent of fibrous pleurisy. The invasion of chest wall adipose tissue by spindled tumour cells is seen at the bottom of the field. (B) Higher-power view of (A) showing fascicular arrays of hyalinised collagen amid which mildly atypical spindle cells infiltrate fat. (C) Sarcomatoid region of a desmoplastic malignant mesothelioma showing diffuse invasion of chest wall skeletal muscle. (D) Desmoplastic malignant mesothelioma invading pulmonary parenchyma. In this example, tumour spreads into the lung as intra-alveolar plugs in a peculiar bronchiolitis obliterans-organising pneumonia-like pattern. (E) Pancytokeratin staining of the tumour in (A) highlights invading tumour cells within adipose tissue of the chest wall. 


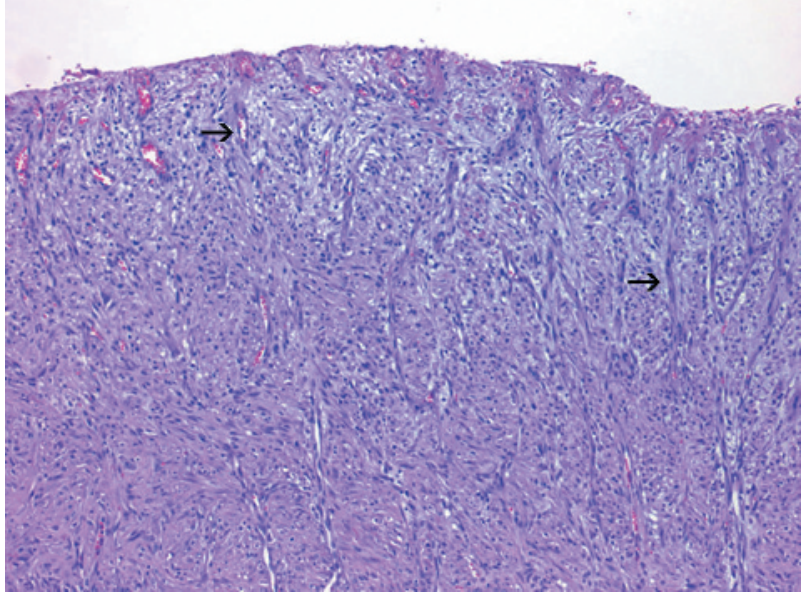

Figure 14 Organising pleuritis showing chronic inflammation and capillaries (arrows) oriented perpendicularly to the pleural surface.

adipose tissue or skeletal muscle or lung, and distant metastases (fig 13)..$^{80}$

\section{Immunohistochemical findings}

Traditional mesothelial markers do not typically stain DMM. The opposite problem is seen with pan-cytokeratin, which stains both neoplastic mesothelial cells in DMM and entrapped reactive mesothelial cells in fibrous pleuritis. The utility of immunohistochemistry analyses in the diagnosis of DMM instead lies in ability of pan-cytokeratin to highlight invasive tumour cells within pulmonary parenchyma or chest wall skeletal muscle or adipose tissue (fig 13).

\section{Differential considerations}

Adherence to the four previously mentioned criteria permits separation of DMM from its main differential consideration, chronic fibrous pleuritis, in most cases. Other features that may aid in the distinction include elongated capillaries perpendicular to the pleural surface and zonation, both of which are seen in reactive pleuritis but not in DMM (fig 14). ${ }^{80}$ Zonation refers to the phenomenon in which the degree of cellularity and atypia is highest near the serosal surface and trails off toward the chest wall.

Desmoplastic malignant mesothelioma can occasionally show basketweave-like areas reminiscent of pleural plaque. Storiform growth, which is common in DMM, is not a feature in pleural plaque. ${ }^{39}$

Localised fibrous tumour of the pleura, with its whorls and haphazard arrays of keloid-like collagen, may be potentially confused with DMM, particularly in small biopsy specimens. Localised fibrous tumour, however, typically has a distinctive pedunculated appearance and, contrary to DMM, is usually CD34 positive and cytokeratin negative. ${ }^{81}$ The rare entity known as calcifying fibrous pseudotumour of the pleura also shows densely hyalinised collagen, but unlike DMM, additionally features psammomatous or dystrophic calcifications. ${ }^{82}$

\section{OTHER PATTERNS}

Several unusual forms of malignant mesothelioma have been described. Small-cell malignant mesothelioma bears a resemblance to small cell carcinoma and other small round cell tumours, such as primitive neuroectodermal tumour (Askin's tumour) and lymphoma. ${ }^{83}{ }^{84}$ A targeted immunohistochemical approach using markers such as TTF-1, LCA and CD99 may aid in the distinction.

Lymphohistiocytoid malignant mesothelioma features large histiocytoid cells with moderately abundant pale
Take-home messages

- Given the histological overlap between malignant mesothelioma and other malignancies that involve the serosal membranes, the use of ancillary techniques such as immunohistochemistry is warranted.

- As there is no single uniformly sensitive and specific immunomarker for malignant mesothelioma, it is a good practice to use a panel of immunostains that includes both mesothelial-associated and carcinomaassociated markers.

- Desmoplastic malignant mesothelioma can be deceptively bland. Foci of sarcomatoid growth, bland necrosis, invasion of chest wall tissue and a lack of zonation are features that help to distinguish desmoplastic malignant mesothelioma from reactive pleuritis.

- Because of the therapeutic, prognostic and potential medicolegal implications of a diagnosis of malignant mesothelioma, a conservative approach is best, especially when interpreting small biopsies.

cytoplasm and a dense accompanying lymphoplasmytic infiltrate. ${ }^{85-87}$ Although this entity may be confused with lymphoma and inflammatory pseudotumour, careful examination will usually show cytokeratin-positive sarcomatoid tumour cells in the background or areas more typical of SMM.

\section{CAVEATS TO THE DIAGNOSIS OF MESOTHELIAL LESIONS}

With the availability of immunostains, the problem in evaluating cytological specimens lies not so much in the distinction between malignant mesothelioma and metastatic carcinoma but in the separation of malignant mesothelioma from reactive mesothelial proliferations. As there is significant overlap between the cytological appearance of reactive and neoplastic mesothelium, it remains my practice, and that of a number of others, not to render a diagnosis of malignant mesothelioma solely on the basis of cytology specimens. ${ }^{39} 88$

Given the prognostic and potential medicolegal implications of a diagnosis of malignant mesothelioma, a lack of certainty as to the diagnosis on small biopsy specimens should prompt a request for a larger tissue sample, preferably one which allows for assessment of invasion of subjacent tissues, such as chest wall or omental fat. A small percentage of tumours remain indeterminate even after extensive examination with ancillary techniques. In most cases, however, by using an integrated approach of clinicoradiographic, histopathological and ancillary techniques, the distinction between malignant mesothelioma and its mimics can be made with confidence.

\section{REFERENCES}

1 Ordonez NG. The diagnostic utility of immunohistochemistry and electron microscopy in distinguishing between peritoneal mesotheliomas and serous carcinomas: a comparative study. Mod Pathol 2006;26:96-100.

2 Baker PM, Clement PB, Young RH. Malignant peritoneal mesothelioma in women: a study of 75 cases with emphasis on their morphologic spectrum and differential diagnosis. Am J Clin Pathol 2005;123:724-37.

3 Ordonez NG. Value of cytokeratin 5/6 immunostaining in distinguishing epithelial mesothelioma of the pleura from lung adenocarcinoma. Am J Surg Pathol 1998;22:1215-21

4 Cury PM, Butcher DN, Fisher C, et al. Value of the mesothelium-associated antibodies thrombomodulin, cytokeratin $5 / 6$, calretinin, and $\mathrm{CD} 44 \mathrm{H}$ in distinguishing epithelioid pleural mesothelioma from adenocarcinoma metastatic to the pleura. Mod Pathol 2000;13:107-12

5 Chu PG, Weiss LM. Expression of cytokeratin 5/6 in epithelial neoplasms: an immunohistochemical study of 509 cases. Mod Pathol 2002;15:6-10. 
6 Ordonez NG. Value of calretinin immunostaining in differentiating epithelial mesothelioma from lung adenocarcinoma. Mod Pathol 1998; 11:929-33.

7 Granville LA, Younes M, Churg A, et al. Comparison of monoclonal versus polyclonal calretinin antibodies for immunohistochemical diagnosis of malignant mesothelioma. Appl Immunohistochem Mol Morphol 2005; 13:75-9.

8 Leers MP, Aarts MM, Theunissen PH. E-cadherin and calretinin: a useful combination of immunochemical markers for differentiation between mesothelioma and metastatic adenocarcinoma. Histopathology 1998;32:209-16.

9 Doglioni C, Dei Tos AP, Laurino L, et al. Calretinin: a novel immunocytochemical marker for mesothelioma. Am J Surg Pathol 1996;20:1037-46.

10 Riera JR, Astengo-Osuna C, Longmate JA, et al. The immunohistochemical diagnostic panel for epithelial mesothelioma: a reevaluation after heatinduced epitope retrieval. Am J Surg Pathol 1997;21:1409-19.

11 Ordonez NG. The immunohistochemical diagnosis of epithelial mesothelioma. Hum Pathol 1999;30:313-23.

12 Corson JM, Pinkus GS. Mesothelioma: profile of keratin proteins and carcinoembryonic antigen: an immunoperoxidase study of 20 cases and comparison with pulmonary adenocarcinomas. Am J Pathol 1982;108:80-7.

13 Di Loreto C, Puglisi F, Di Lauro V, et al. TTF-1 protein expression in pleural malignant mesotheliomas and adenocarcinomas of the lung. Cancer Lett 1998;124:73-8.

14 Ordonez NG. Value of thyroid transcription factor-1, E-cadherin, BG8, WT1, and CD44S immunostaining in distinguishing epithelial pleural mesothelioma from pulmonary and nonpulmonary adenocarcinoma. Am J Surg Pathol 2000;24:598-606.

15 Bejarano PA, Mousavi F. Incidence and significance of cytoplasmic thyroid transcription factor-1 immunoreactivity. Arch Pathol Lab Med 2003;127:193-5

16 Ordonez NG. Role of immunohistochemistry in distinguishing epithelial peritoneal mesotheliomas from peritoneal and ovarian serous carcinomas. Am J Surg Pathol 1998;22:1203-14.

17 Ordonez NG. Value of the Ber-EP4 antibody in differentiating epithelial pleural mesothelioma from adenocarcinoma. The M.D. Anderson experience and a critical review of the literature. Am J Clin Pathol 1998;109:85-9.

18 Garcia-Prats MD, Ballestin C, Sotelo T, et al. A comparative evaluation of immunohistochemical markers for the differential diagnosis of malignant pleural tumours. Histopathology 1998;32:462-72.

19 Chu AY, Litzky LA, Pasha TL, et al. Utility of D2-40, a novel mesothelial marker, in the diagnosis of malignant mesothelioma. Mod Pathol 2005; 18:105-10

20 Ordonez NG. D2-40 and podoplanin are highly specific and sensitive immunohistochemical markers of epithelioid malignant mesothelioma. Hum Pathol 2005;36:372-80

21 Schacht V, Dadras SS, Johnson LA, et al. Up-regulation of the lymphatic marker podoplanin, a mucin-type transmembrane glycoprotein, in human squamous cell carcinomas and germ cell tumors. Am J Pathol 2005;166:913-21

22 Gulyas M, Hjerpe A. Proteoglycans and WT1 as markers for distinguishing adenocarcinoma, epithelioid mesothelioma, and benign mesothelium. J Pathol 2003; 199:479-87.

23 Foster MR, Johnson JE, Olson SJ, et al. Immunohistochemical analysis of nuclear versus cytoplasmic staining of WT1 in malignant mesotheliomas and primary pulmonary adenocarcinomas. Arch Pathol Lab Med 2001;125:1316-20.

24 Oates J, Edwards C. HBME-1, MOC-31, WT1 and calretinin: an assessment of recently described markers for mesothelioma and adenocarcinoma. Histopathology 2000;36:341-7.

25 Campbell CE, Kuriyan NP, Rackley RR, et al. Constitutive expression of the Wilms tumor suppressor gene (WT1) in renal cell carcinoma. Int J Cancer 1998;78:182-8.

26 Dupont J, Wang X, Marshall DS, et al. Wilms tumor gene (WT1) and p53 expression in endometrial carcinomas: a study of 130 cases using a tissue microarray. Gynecol Oncol 2004;94:449-55.

27 Goldstein NS, Bassi D, Uzieblo A. WT1 is an integral component of an antibody panel to distinguish pancreaticobiliary and some ovarian epithelial neoplasms. Am J Clin Pathol 2001;116:246-52.

28 Miettinen M, Sarlomo-Rikala M. Expression of calretinin, thrombomodulin, keratin 5, and mesothelin in lung carcinomas of different types: an immunohistochemical analysis of 596 tumors in comparison with epithelioid mesotheliomas of the pleura. Am J Surg Pathol 2003;27:150-8.

29 Ordonez NG. The diagnostic utility of immunohistochemistry in distinguishing between mesothelioma and renal cell carcinoma: a comparative study. Hum Pathol 2004;35:697-710.

30 Ordonez NG. Value of thrombomodulin immunostaining in the diagnosis of mesothelioma. Histopathology 1997;31:25-30.

31 Frierson HF Jr, Moskaluk CA, Powell SM, et al. Large-scale molecular and tissue microarray analysis of mesothelin expression in common human carcinomas. Hum Pathol 2003;34:605-9.

32 Ordonez NG. The value of antibodies 44-3A6, SM3, HBME-1, and thrombomodulin in differentiating epithelial pleural mesothelioma from lung adenocarcinoma: a comparative study with other commonly used antibodies. Am J Surg Pathol 1997;21:1399-408.

33 Ordonez NG. Value of mesothelin immunostaining in the diagnosis of mesothelioma. Mod Pathol 2003;16:192-7.

34 Ordonez NG. Value of the MOC-31 monoclonal antibody in differentiating epithelial pleural mesothelioma from lung adenocarcinoma. Hum Pathol 1998;29:166-9.
35 Roberts F, Harper CM, Downie I, et al. Immunohistochemical analysis still has a limited role in the diagnosis of malignant mesothelioma. A study of thirteen antibodies. Am J Clin Pathol 2001;1 16:253-62.

36 Wick MR, Loy T, Mills SE, et al. Malignant epithelioid pleural mesothelioma versus peripheral pulmonary adenocarcinoma: a histochemical, ultrastructural, and immunohistologic study of 103 cases. Hum Pathol 1990;21:759-66.

37 Roggli VL, Kolbeck J, Sanfilippo F, et al. Pathology of human mesothelioma. Etiologic and diagnostic considerations. Pathol Annu 1987;22(2 Pt):91-131.

38 Coleman M, Henderson DW, Mukherjee TM. The ultrastructural pathology of malignant pleural mesothelioma. Pathol Annu 1989;24(1 Pt):303-53.

39 Sporn TA, Roggli VL. Mesothelioma. In: Roggli VL, Oury TD, Sporn TA, eds. Pathology of asbestos-associated disease.2nd edn. New York: Springer, 2004:104-68.

40 Dardick I, Jabi M, McCaughey WT, et al. Diffuse epithelial mesothelioma: a review of the ultrastructural spectrum. Ultrastruct Pathol 1987;11:503-33.

41 Del Frate C, Mortele K, Zanardi R, et al. Pseudomesotheliomatous angiosarcoma of the chest wall and pleura. J Thorac Imaging 2003; 18:200-3

42 Attanoos RL, Gibbs AR. 'Pseudomesotheliomatous' carcinomas of the pleura: a 10-year analysis of cases from the Environmental Lung Disease Research Group, Cardiff. Histopathology 2003;43:444-52.

43 Harwood TR, Gracey DR, Yokoo H. Pseudomesotheliomatous carcinoma of the lung. A variant of peripheral lung cancer. Am J Clin Pathol 1976;65:159-67.

44 Crotty TB, Myers JL, Katzenstein AL, et al. Localized malignant mesothelioma. A clinicopathologic and flow cytometric study. Am J Surg Pathol 1994; 18:357-63.

45 Allen TC, Cagle PT, Churg AM, et al. Localized malignant mesothelioma Am J Surg Pathol 2005;29:866-73.

46 Dessy $E$, Falleni $M$, Braidotti $P$, et al. Unusual clear cell variant of epithelioid mesothelioma. Arch Pathol Lab Med 2001;125:1588-90.

47 Osborn M, Pelling N, Walker MM, et al. The value of 'mesotheliumassociated' antibodies in distinguishing between metastatic renal cell carcinomas and mesotheliomas. Histopathology 2002;41:301-7.

48 Ordonez NG. Mesothelioma with clear cell features: an ultrastructural and immunohistochemical study of 20 cases. Hum Pathol 2005;36:465-73.

49 Nascimento AG, Keeney GL, Fletcher CD. Deciduoid peritoneal mesothelioma. An unusual phenotype affecting young females. Am J Surg Pathol 1994; 18:439-45.

50 Fan Z, van de Rijn M, Montgomery K, et al. Hep par 1 antibody stain for the differential diagnosis of hepatocellular carcinoma: 676 tumors tested using tissue microarrays and conventional tissue sections. Mod Pathol 2003;16:137-44.

51 Galateau-Salle F, Vignaud JM, Burke L, et al. Well-differentiated papillary mesothelioma of the pleura: a series of 24 cases. Am J Surg Pathol 2004;28:534-40

52 Butnor KJ, Sporn TA, Hammar SP, et al. Well-differentiated papillary mesothelioma. Am J Surg Pathol 2001;25:1304-9.

53 Daya D, McCaughey WT. Well-differentiated papillary mesothelioma of the peritoneum. A clinicopathologic study of 22 cases. Cancer 1990;65:292-6.

54 Churg A, Roggli VL, Galateau-Salle F, et al. Mesothelioma. In: Travis WD, Brambilla E, Muller-Hermelink HK, Harris CC, eds. World Health Organization classification of tumours. Pathology and genetics of tumours of the lung, pleura, thymus, and heart. Lyon: IARC Press, 2004:128-36.

55 Goldblum J, Hart WR. Localized and diffuse mesotheliomas of the genital tract and peritoneum in women. A clinicopathologic study of nineteen true mesothelial neoplasms, other than adenomatoid tumors, multicystic mesotheliomas, and localized fibrous tumors. Am J Surg Pathol 1995; 19:1124-37.

56 Churg A, Colby TV, Cagle P, et al. The separation of benign and malignant mesothelial proliferations. Am J Surg Pathol 2000;24:1 183-200.

57 Ordonez NG, Ro JY, Ayala AG. Lesions described as nodular mesothelial hyperplasia are primarily composed of histiocytes. Am J Surg Pathol 1998;22:285-92

58 Attanoos RL, Griffin A, Gibbs AR. The use of immunohistochemistry in distinguishing reactive from neoplastic mesothelium. A novel use for desmin and comparative evaluation with epithelial membrane antigen, p53, plateletderived growth factor-receptor, P-glycoprotein and $\mathrm{Bcl}-2$. Histopathology 2003;43:231-8

59 Wolanski KD, Whitaker D, Shilkin KB, et al. The use of epithelial membrane antigen and silver-stained nucleolar organizer regions testing in the differential diagnosis of mesothelioma from benign reactive mesothelioses. Cancer 1998:82:583-90.

60 Ross MJ, Welch WR, Scully RE. Multilocular peritoneal inclusion cysts (socalled cystic mesotheliomas). Cancer 1989;64:1336-46.

61 Attanoos RL, Galateau-Salle F, Gibbs AR, et al. Primary thymic epithelial tumours of the pleura mimicking malignant mesothelioma. Histopathology 2002;41:42-9.

62 Vaideeswar P, Deshpande JR, Jambhekar NA. Primary pleuro-pulmonary malignant germ cell tumours. J Postgrad Med 2002;48:29-31.

63 DeYoung BR, Wick MR. Immunohistologic evaluation of metastatic carcinomas of unknown origin: an algorithmic approach. Semin Diagn Pathol 2000;17:184-93.

64 Zhang PJ, Livolsi VA, Brooks JJ. Malignant epithelioid vascular tumors of the pleura: report of a series and literature review. Hum Pathol 2000;31:29-34.

65 Crotty EJ, McAdams HP, Erasmus JJ, et al. Epithelioid hemangioendothelioma of the pleura: clinical and radiologic features. Am J Roentgenol 2000; 175:1545-9. 
66 Battifora H, McCaughey WTE. Diffuse malignant mesothelioma. In: Rosai J, Sobin LH, eds. Atlas of tumor pathology: tumors of the serosal membranes. Washington, DC: Armed Forces Institute of Pathology, 1995: 17-84.

67 Andrion A, Mollo F. Leiomyoid mesotheliomas. Histopathology 1993;22:601-2.

68 Mayall FG, Goddard H, Gibbs AR. Intermediate filament expression in mesotheliomas: leiomyoid mesotheliomas are not uncommon. Histopathology 1992;21:453-7.

69 Yousem SA, Hochholzer L. Malignant mesotheliomas with osseous and cartilaginous differentiation. Arch Pathol Lab Med 1987; 11 1:62-6.

70 Lucas DR, Pass HI, Madan SK, et al. Sarcomatoid mesothelioma and its histological mimics: a comparative immunohistochemical study. Histopathology 2003:42:270-9

71 Attanoos RL, Dojcinov SD, Webb R, et al. Anti-mesothelial markers in sarcomatoid mesothelioma and other spindle cell neoplasms. Histopathology 2000;37:224-31

72 Hurlimann J. Desmin and neural marker expression in mesothelial cells and mesotheliomas. Hum Pathol 1994;25:753-7.

73 Bueno R, Reblando J, Glickman J, et al. Pleural biopsy: a reliable method for determining the diagnosis but not subtype in mesothelioma. Ann Thorac Surg 2004;78: 1774-6

74 Miettinen M, Limon J, Niezabitowski A, et al. Calretinin and other mesothelioma markers in synovial sarcoma: analysis of antigenic similarities and differences with malignant mesothelioma. Am J Surg Pathol $2001 ; 25: 610-7$.

75 Carbone M, Rizzo P, Powers A, et al. Molecular analyses, morphology and immunohistochemistry together differentiate pleural synovial sarcomas from mesotheliomas: clinical implications. Anticancer Res 2002;22:3443-8.

76 Wilson GE, Hasleton PS, Chatterjee AK. Desmoplastic malignant mesothelioma: a review of 17 cases. J Clin Pathol 1992;45:295-8.

77 Cantin R, Al-Jabi M, McCaughey WT. Desmoplastic diffuse mesothelioma. Am J Surg Pathol 1982;6:215-22.
78 Kannerstein $M$, Churg J. Desmoplastic diffuse malignant mesothelioma. Prog Surg Pathol 1980;1:19-27.

79 Colby TV. Malignancies in the lung and pleura mimicking benign processes. Semin Diagn Pathol 1995; 12:30-44.

80 Mangano WE, Cagle PT, Churg A, et al. The diagnosis of desmoplastic malignant mesothelioma and its distinction from fibrous pleurisy: a histologic and immunohistochemical analysis of 31 cases including p53 immunostaining. Am J Clin Pathol 1998;110:191-9.

81 Flint A, Weiss SW. CD-34 and keratin expression distinguishes solitary fibrous tumor (fibrous mesothelioma) of pleura from desmoplastic mesothelioma. Hum Pathol 1995;26:428-31.

82 Pinkard NB, Wilson RW, Lawless N, et al. Calcifying fibrous pseudotumor of pleura. A report of three cases of a newly described entity involving the pleura. Am J Clin Pathol 1996; 105:189-94.

83 Falconieri G, Zanconati F, Bussani R, et al. Small cell carcinoma of lung simulating pleural mesothelioma. Report of 4 cases with autopsy confirmation. Pathol Res Pract 1995;191:1147-52.

84 Mayall FG, Gibbs AR. The histology and immunohistochemistry of small cell mesothelioma. Histopathology 1992;20:47-51.

85 Yao DX, Shia J, Erlandson RA, et al. Lymphohistiocytoid mesothelioma: a clinical, immunohistochemical and ultrastructural study of four cases and literature review. Ultrastruct Pathol 2004;28:213-28.

86 Khalidi HS, Medeiros $\amalg$, Battifora H. Lymphohistiocytoid mesothelioma. An often misdiagnosed variant of sarcomatoid malignant mesothelioma. Am J Clin Pathol 2000;113:649-54.

87 Henderson DW, Attwood HD, Constance TJ, et al. Lymphohistiocytoid mesothelioma: a rare lymphomatoid variant of predominantly sarcomatoid mesothelioma. Ultrastruct Pathol 1988;12:367-84.

88 Renshaw AA, Dean BR, Antman KH, et al. The role of cytologic evaluation of pleural fluid in the diagnosis of malignant mesothelioma. Chest 1997;111:106-9. 